\title{
Avaliação de Métodos de Exigência de Capital para Risco de Ações no Brasil
}

\author{
Gustavo Silva Araújo \\ João Maurício de Souza Moreira \\ Ricardo dos Santos Maia Clemente
}

\section{Resumo}

Este trabalho examina quatro métodos de determinação da exigência de capital para cobertura de risco de mercado de instituições financeiras, decorrente da exposição em ações e seus derivativos, excetuando-se o caso de opções. Para as simulações são montadas duas carteiras com ativos que compõem o Ibovespa. Os métodos avaliados seguem as orientações do Comitê de Basiléia, inserindose o primeiro método na abordagem padronizada e os demais na de modelos internos, que utilizam o conceito de Valor em Risco (VaR). Os métodos de VaR empregados são: histórico, diagonal e alisamento exponencial (Riskmetrics ${ }^{\mathrm{TM}}$ ). A aferição destes métodos segue metodologia indicada por Basiléia, que se baseia no VaR para o horizonte de 1 dia. Adicionalmente, aplica-se o teste de Kupiec para proporção de exceções, verifica-se o número de observações que ultrapassam a exigência de capital para cada método, bem como a magnitude das diferenças entre o capital exigido e as perdas verificadas. Embora o método histórico tenha apresentado um melhor desempenho do VaR, os melhores resultados no tocante à exigência de capital são obtidos pelo método de alisamento exponencial.

Palavras-chave: exigência de capital; risco de mercado; VaR; acordo de Basiléia.

\begin{abstract}
This paper analyses four methods of calculating capital requirements for coverage of market risk due to exposure in stocks and their derivatives, except options. For simulation purposes, two theoretical portfolios are created with assets that participate in the composition of Ibovespa. The methods evaluated follow the directives of the Basel Committee. The first is based on the standardized approach and the others, on the internal models approach based on the Value-atRisk (VaR) concept. The VaR methods evaluated are historical, Diagonal and the Riskmetrics ${ }^{\mathrm{TM}}$ approach. The backtesting of the methods follows the methodology suggested by the Committee. Additionally, for the methods based on VaR, it is applied the Kupiec test for proportion of failures. The relevance of the difference between the capital required and the verified losses is also considered. Although the historical method presents the best VaR performance, the best results with respect to the capital requirements belong to the Riskmetrics ${ }^{\mathrm{TM}}$ approach.
\end{abstract}

Key words: capital requirement; market risk; VaR; Basel capital accord. 


\section{INTRODUÇÃO}

O Comitê da Basiléia elaborou um conjunto de propostas que procura fornecer às autoridades de supervisão bancária nacionais as diretrizes básicas para a regulamentação da exigência de capital (EC) para cobertura do risco de mercado. As propostas sugerem, para efeito de alocação de capital, duas alternativas de cálculo: a abordagem padronizada e a abordagem baseada em modelos internos de gestão de risco.

A abordagem padronizada apresenta algumas limitações na metodologia de cálculo da EC, como, por exemplo, sua natureza estática, o que a torna inadequada para capturar alterações nos padrões de volatilidade e nas correlações dos fatores de risco. Esta limitação é preocupante, principalmente em se tratando de mercados sujeitos a oscilações consideráveis nos preços de seus ativos. A EC baseada em tal método, ao ser calibrada para determinado cenário, pode tornarse excessiva, caso haja uma redução nos níveis de volatilidade, ou insuficiente em caso contrário.

A abordagem de modelos internos, baseada no conceito de valor em risco (VaR), teve como ponto de partida o consenso de que o cálculo do capital regulamentar deveria ser sensível ao perfil de risco corrente e de que as instituições financeiras seriam capazes de elaborar modelos mais apurados, por terem maior conhecimento das carteiras que administram. A gestão ativa destes modelos proporcionaria maior eficiência na alocação de capital, em comparação à predeterminação de percentuais aplicados sobre os ativos das instituições e indistintamente adotados por todo o sistema financeiro. Com a finalidade de assegurar um grau mínimo de padronização, transparência e consistência dos resultados obtidos para os diferentes sistemas proprietários, são estabelecidas algumas restrições qualitativas e quantitativas, destacando-se entre estas últimas o horizonte de investimento (holding period) de dez dias, o nível de segurança de $99 \%$ e o multiplicador mínimo de três ${ }^{(1)}$.

Hendricks e Hirtle (1997) enfatizam as vantagens de se utilizar modelos internos para o cálculo de exigência de capital, com destaque para a característica dinâmica do VaR, que permite rápida adaptação às alterações nos padrões de volatilidade. Crouhy, Galai e Mark (1998) comparam a abordagem de modelos internos à padronizada de Basiléia para diversas carteiras e constatam uma considerável redução no capital regulamentar requerido. Amante e Lemgruber (2001) comparam as duas abordagens no mercado acionário brasileiro e concluem 
que a utilização da abordagem interna traz níveis de provisionamento adequados à exposição ao risco de mercado das carteiras estudadas, enquanto a exigência de capital requerida pelo modelo padronizado mostra-se inadequada para os altos padrões de volatilidade do mercado brasileiro.

A autoridade monetária brasileira vem optando por adotar modelos padronizados de exigência de capital para os fatores de risco de mercado(2). Já estão contemplados os fatores câmbio e taxa de juros prefixada em reais, sendo que este último é um modelo padronizado com características de modelo interno, uma vez que utiliza o VaR e, por conseqüência, considera alterações na volatilidade do mercado. Desta forma, o sistema financeiro brasileiro ainda carece de normativos sobre exigência de capital para risco de mercado de outros fatores, entre os quais figuram as ações destinadas à negociação (i.e. do trading book).

Este trabalho busca avaliar quatro métodos de determinação da EC, na linha preconizada pelo Comitê de Basiléia, para a cobertura de risco de mercado em ações no Brasil. O primeiro método segue a abordagem padronizada, enquanto os demais se inserem na abordagem de modelos internos. Estes últimos utilizam o conceito de VaR e apresentam a vantagem de se adaptarem a oscilações nas volatilidades e nas correlações entre os ativos.

\section{Amostra e Metodologia}

São utilizadas as séries de cotações do Índice da Bolsa de Valores de São Paulo - Ibovespa - e dos preços de dez das ações que o compõem (ver Tabela 1), registrados no fechamento do pregão no período de 4/7/1994 a 31/7/2002, bem como a série de preços de fechamento da Telebrás no período de 4/7/1994 a 14/ 10/1999, obtidas no sistema de informações Economática com ajuste para pagamento de proventos. Algumas destas ações não apresentam a mesma liquidez no início do período em estudo, não havendo cotações registradas para alguns dias da amostra. Nestes casos opta-se por repetir o valor da última cotação registrada. Uma situação especial envolve o uso das séries de Recibo de Telebrás RPN e Telemar PN. A série relativa à empresa Telemar inicia-se somente a partir de 29/9/98, mas como esta ação tem participado da composição do Ibovespa com o maior percentual dentre todos os papéis, constituindo-se em ativo com grandes volumes negociados na bolsa desde então, não seria adequado excluí-la da análise ${ }^{(3)}$. Em situação análoga encontrava-se a Telebrás no período anterior. Assim, nas carteiras construídas, o Recibo de Telebrás ocupa o lugar da Telemar PN durante o período em que esta ainda não era negociada ${ }^{(4)}$. 


\section{Tabela 1: Composição das Carteiras Utilizadas nas Avaliações de Métodos de Exigência de Capital para Ações}

\begin{tabular}{|c|c|}
\hline Carteira & Composição \\
\hline I & $\begin{array}{l}(+) \mathrm{ARCZ6},(+) \mathrm{BBDC} 4,(+) \mathrm{CMIG} 4,(+) \mathrm{CSNA3},(+) \text { ELET6, }(+) \mathrm{INEP} 4,(+) \\
\text { ITAU4, (+)PETR4, (+)RCTB41/TNLP4, (+)VALE5 }\end{array}$ \\
\hline II & $\begin{array}{l}\text { (-)ARCZ6, (+)BBDC4, (+)CMIG4, (-)CSNA3, (+)ELET6, (-) INEP4, } \\
\text { (+)ITAU4, (-)PETR4, (+)RCTB41/TNLP4, (+)VALE5 }\end{array}$ \\
\hline
\end{tabular}

Obs: o sinal de (+) ou (-) indica que a posição do ativo na carteira em questão é, respectivamente, comprada ou vendida. Em ambas as carteiras o período considerado para aferição dos métodos é de 19/10/1995 a 31/7/2002. A Telebrás compõe ambas as carteiras até 29/9/99, a partir de quando é substituída pela Telemar. Os ativos acima identificados pelos respectivos códigos na Bovespa referem-se às empresas: Aracruz, Bradesco, CEMIG, CSN, Eletrobrás, Inepar, Itaú, Petrobrás, Telebrás/Telemar e Vale do Rio Doce.

Desta forma, cada série de cotações de ações apresenta 1.996 observações, o mesmo ocorrendo para a série composta Telebrás/Telemar. São construídas duas carteiras, contendo dez ativos cada, tal que o valor de cada carteira é constante ao longo do período estudado e a proporção $\mathrm{w}_{\mathrm{i}}$ do valor financeiro com que cada ação participa em uma dada carteira é constante e igual em módulo para todas as ações.

O critério básico para a definição das duas carteiras, neste trabalho, é a liquidez dos ativos componentes. Dentre as ações que compunham o Ibovespa na época, são selecionados os papéis com menor número de falhas na série de cotações, restringindo-se ao máximo de duas empresas de um mesmo setor. Neste sentido, procura-se, para ambas as carteiras, conferir um grau intermediário de diversificação, considerando-se que tanto mais diversificada seria uma carteira quanto maior o número de ações que a compõem, quanto menor a concentração de ações de um mesmo setor e quanto mais expressiva a participação de tais ações na composição do Ibovespa ${ }^{(5)}$. Busca-se ainda variar a composição das carteiras apenas com relação à existência de posições somente compradas ou posições compradas e vendidas. As duas carteiras utilizadas encontram-se na Tabela 1. Em ambas as carteiras atribui-se o valor de $\mathrm{R} \$ 10.000,00$ a cada posição. Desta forma, a Carteira I monta a R \$ 100.000,00, uma vez que todas as posições são compradas. Já a Carteira II, por possuir seis posições compradas e quatro vendidas, detém o montante de $\mathrm{R} \$ 20.000,00$.

A utilização de apenas duas carteiras leva em conta que o objetivo precípuo do trabalho é a avaliação comparativa do desempenho dos métodos mais conhecidos de VaR, quando utilizados para a determinação de requerimento regulamentar de capital para risco de mercado de instituições financeiras, principalmente quando contrapostos a métodos baseados na abordagem padronizada, que são insensíveis 
ao risco. Como o VaR é função direta da volatilidade dos ativos considerados, admite-se que outras composições de carteira terão, ainda que não perfeitamente, seus patamares de risco refletidos no capital requerido por modelos internos.

Vale observar que as posições em derivativos de ações, excetuando-se o caso das opções, podem ser tratadas, no que tange ao risco de mercado relativo ao fator de risco preço das ações, como posições no mercado a vista. O risco de taxa de juros envolvido deve ser tratado em separado no âmbito do fator de risco taxa de juros.

\section{Cálculo das Séries de Retornos}

O retorno de um dia é dado por $R_{t}=\left(p_{t} / p_{t-1}\right)-1$, onde $\mathrm{p}_{\mathrm{t}}$ é o preço de fechamento da ação na data t. Analogamente, para dez dias, o retorno é dado por $R_{t}=\left(p_{t} / p_{t-10}\right)-1$. Para os métodos paramétricos de cálculo do VaR, utiliza-se a aproximação do retorno de um dia pela forma logarítmica, $r_{t}=\ln \left(p_{t} / p_{t-1}\right)$, onde ln denota o logaritmo neperiano. A opção por esta aproximação decorre do melhor ajustamento à distribuição normal da série logarítmica em comparação à série usual.

\section{Métodos de Determinação da Exigência de Capital}

São avaliados quatro métodos de determinação da exigência de capital (EC) para fazer face ao risco de mercado incorrido por posições assumidas em ações. O primeiro método segue a abordagem padronizada e se caracteriza por uma EC constante ao longo do tempo. Os demais se inserem na abordagem de modelos internos, nos termos do Acordo de Basiléia, cuja EC é diariamente calculada por:

$$
E C_{t}=\operatorname{máx}\left\{\left(\frac{M}{60} \sum_{k=1}^{60} V a R_{t-k+1}\right), V a R_{t}\right\},
$$

onde $\mathrm{M}$ = 3 é o multiplicador da média de $\mathrm{VaR}_{\mathrm{t}}$ nos últimos 60 dias. Considerando que a volatilidade no mercado acionário brasileiro é consideravelmente alta e que isto aliado a $M=3$ pode levar a exigências de capital exageradas em alguns períodos, avalia-se adicionalmente a $\mathrm{EC}$ calculada com $\mathrm{M}=2 . \mathrm{VaR}_{\mathrm{t}}$ é o valor em risco na data $t$, em unidades monetárias, associado a uma dada carteira de ações. $\mathrm{O} \mathrm{VaR}_{\mathrm{t}}$ é calculado para um horizonte de dez dias. O nível de confiança exigido é de $99 \%$, ou seja, o nível pré-especificado para o valor em risco é de $1 \%$. 


\section{Método da Abordagem Padronizada}

Neste método são considerados dois tipos de risco: específico e geral. O risco específico está associado a cada ação em particular, independentemente do que ocorre com o mercado. É o risco diversificável ou não sistemático. O risco geral está associado ao contexto do mercado, quando todos os papéis estão sujeitos, em maior ou menor intensidade, à influência de fatores macroeconômicos. É o risco não diversificável ou sistemático.

A base para o cálculo da exigência de capital para risco específico é a soma dos módulos das posições compradas e vendidas. No caso do risco geral, a base de cálculo é dada pelo módulo da soma de posições compradas e vendidas. Sobre cada uma destas bases são aplicados, alternativamente, os percentuais de $8 \%, 12 \%$ e $15 \%$. A EC para cobertura de risco de mercado para ações é dada pela soma desses dois montantes. Desta forma, para a Carteira I, os valores de exigência de capital examinados são, respectivamente, $R \$ 16.000,00, R \$ 24.000,00$ e R \$30.000,00. Já para a Carteira II, os montantes são, respectivamente, $\mathrm{R} \$ 9.600,00, \mathrm{R} \$ 14.400,00$ e $\mathrm{R} \$ 18.000,00$.

\section{Método Diagonal}

Este método utiliza o VaR paramétrico, com base na distribuição normal padronizada, calculado para o prazo de um dia pela fórmula ${ }^{(6)}$ :

$$
\operatorname{VaR}_{t}^{1 d}=\left|V_{c, t} \times z_{\alpha \%} \times \sigma_{t}\right|
$$

onde $\mathrm{V}_{\mathrm{c}, \mathrm{t}}$ é o valor financeiro da carteira em $\mathrm{t}, \mathrm{z}_{\alpha \%}$ é o quantil da distribuição normal padronizada relativo ao percentil $\alpha$ e $\sigma_{\mathrm{t}}$ é o desvio padrão da carteira em t. O Comitê determina um percentil $\alpha$ igual a $1 \%$, de forma que $\mathrm{z}_{\alpha \%}$ é aproximadamente -2,33. O VaR para o intervalo de dez dias para o dia t é obtido por $\operatorname{VaR}_{t}^{10 d}=\operatorname{VaR}_{t-10}^{1 d} \times \sqrt{10}$.

O cálculo de $\sigma_{\mathrm{t}}$ pode tornar-se complexo para um número grande de ativos, na medida em que envolve o uso de uma matriz de covariância (ou correlação) de ordem $\mathrm{n}$ (número de ativos que compõem a carteira). Com efeito, para $\mathrm{n}$ ativos será necessário o cálculo de n(n-1)/2 covariâncias e n variâncias, ou seja, $n(n+1) / 2$ parâmetros. Uma tentativa de tornar mais prático este cálculo reside na utilização dos betas das ações $\left(\beta_{\mathrm{i}}\right)$ para o cálculo da volatilidade da carteira ${ }^{(7)}$. Neste método, usualmente referenciado como diagonal, a variância da carteira é dada $\operatorname{por}^{(8)}$ :

$$
\sigma_{t}^{2}=\sigma_{m, t}^{2} \sum_{i=1}^{n} \sum_{j=1}^{n} w_{i, t} \beta_{i, t} w_{j, t} \beta_{j, t}+\sum_{i=1}^{N} w_{i}^{2} \sigma_{\varepsilon_{i}}^{2}
$$


onde $\sigma_{m, t}^{2}$ é a variância do Ibovespa calculada para o dia t, estimada diariamente pela variância amostral $s_{t}^{2}=\frac{1}{n-1} \sum_{t=1}^{n}\left(r_{t}-\mu_{r}\right)^{2}$, onde $\mu_{\mathrm{r}}$ é a média dos retornos em uma amostra de 252 dias úteis. $\mathrm{E} \sigma_{\varepsilon_{t}}^{2}$ é a variância dos resíduos relativos às regressões dos retornos da ação i sobre os retornos do Ibovespa, realizadas para a obtenção dos respectivos betas. Com este método o volume de cálculo é reduzido sensivelmente, em relação à matriz de covariância tradicional (o número de parâmetros estimados cai para $2 n+1$ ), ao supor que a movimentação conjunta das ações se origina de um único fator comum, o mercado. Os $\beta_{\mathrm{i}, \mathrm{t}}$ são calculados diariamente com base em uma janela móvel de 252 dias úteis. Em vista isso, com relação à descontinuidade das séries Telebrás e Telemar, a Telebrás comporá as carteiras até 29/9/1999, embora a Telemar já fosse negociada há um ano.

Adicionalmente, implementa-se uma forma simplificada, sugerida pelo Comitê da Basiléia para refletir o risco de mercado de carteiras bem diversificadas ${ }^{(9)}$. Nesta aproximação, é desprezado o termo $\sum_{i=1}^{N} w_{i}^{2} \sigma_{\varepsilon_{i}}^{2}$, que tende a se tornar irrelevante à medida que cresce a quantidade de títulos na carteira (ao gerar $\mathrm{w}_{\mathrm{i}}$ pequenos). Embora isto tenda a ocorrer no caso de carteiras com posições apenas compradas, pode não se verificar para carteiras com posições mistas, em que o valor total (líquido) da carteira venha a ser pequeno em relação aos módulos dos montantes de algumas posições, compradas ou vendidas, gerando pesos expressivos para as respectivas ações.

\section{Método Baseado em Alisamento Exponencial}

Este método segue a metodologia do RiskMetrics ${ }^{\mathrm{TM}}$, onde o VaR de cada ativo componente da carteira é obtido a partir da sua volatilidade específica, que é calculada com base em alisamento exponencial (Exponentially Weighted Moving Average - EWMA) ${ }^{(10)}$. Desta forma, o VaR para um dia de cada ativo seria dado por:

$$
\operatorname{VaR}_{i, t}^{1 d}=\left|V_{i, t} \times z_{\alpha \%} \times h_{i, t}\right|
$$

em que $\mathrm{V}_{\mathrm{i}, \mathrm{t}}$ é o valor da posição no ativo i e $\mathrm{h}_{\mathrm{i}, \mathrm{t}}$ é a volatilidade condicional diária dos retornos do ativo i estimada para a data t, calculada por alisamento exponencial, conforme a expressão:

$$
h_{i, t}=\sqrt{\lambda h_{i, t-1}^{2}+(1-\lambda) r_{i, t-1}^{2}}
$$


onde $\lambda$ é o fator de decaimento exponencial, para o qual são usados neste trabalho dois valores. O primeiro, 0,94 , corresponde ao valor adotado pelo RiskMetrics $^{\mathrm{TM}}$. O segundo, 0,90, foi estimado por máxima verossimilhança com base nos retornos do Ibovespa, verificados entre 1/12/1994 e 31/7/2002. Neste procedimento, admite-se a hipótese de retornos normalmente distribuídos. Desta forma, busca-se usar um $\lambda$ que espelhe o mercado acionário brasileiro, visto que a utilização de diferentes fatores de decaimento para os ativos de uma carteira pode inviabilizar o procedimento, ao não garantir que a matriz de covariância destes ativos seja positiva e definida. O VaR de um dia para a carteira de ativos será então

$$
\operatorname{VaR}_{t}^{1 d}=\sqrt{\sum_{i=1}^{n} \sum_{j=1}^{n} \operatorname{VaR}_{i, t}^{1 d} \times \operatorname{VaR} R_{j, t}^{1 d} \times \rho_{(i, j), t}}
$$

onde a correlação entre os ativos i e j na data t, $\rho_{(i, j), t}$, é obtida por $\rho_{(i, j), t}=\frac{h_{(i, j), t}}{h_{i, t} h_{j, t}}$, tal que $\mathrm{h}_{(\mathrm{i}, \mathrm{j}), \mathrm{t}}$ denota a covariância condicional entre os ativos i e j na data t, obtida pela fórmula:

$$
h_{(i, j), t}=\lambda h_{(i, j), t-1}+(1-\lambda) r_{i, t-1} r_{j, t-1}
$$

Estendendo o horizonte para dez dias, o VaR da carteira para o dia t é calculado por:

$$
\operatorname{VaR}_{t}^{10 d}=\operatorname{VaR}_{t-10}^{1 d} \times \sqrt{10}
$$

\section{Método Baseado em VaR Histórico}

Esse método consiste simplesmente em utilizar um dado quantil (aqui, referente ao percentil 1\%) da distribuição empírica dos retornos da carteira, em uma janela móvel de 252 dias úteis, como a estimativa do VaR que comporá o cálculo da exigência de capital ${ }^{(11)}$. O retorno diário da carteira é dado por $R_{c}=\sum_{i} w_{i} \times r_{i}$, onde $\mathrm{r}_{\mathrm{i}}$ é o retorno diário do ativo i. Para cada data $\mathrm{t}$, os pesos $\left(w_{i}\right)$ que ponderam os últimos 252 retornos são os verificados em t, a fim de que a série de retornos reflita a composição da carteira naquela data. Neste trabalho, como a composição das carteiras não muda ao longo do tempo, tal aspecto se verifica automaticamente. O VaR de dez dias, utilizado no cálculo da exigência de capital, é dado pelo produto do VaR de um dia pela raiz quadrada de dez, conforme indicado pelo Comitê da Basiléia. A regra da raiz quadrada pode ser teoricamente justificada para modelos paramétricos que adotem hipóteses tais como retornos normais e i.i.d. Contudo o Comitê de Basiléia não faz nenhuma 
distinção desta ordem, possibilitando o uso da regra para qualquer modelo baseado em $\operatorname{VaR}^{(12)}$. Uma alternativa seria calcular o VaR de dez dias com base na série de retornos de dez dias. Porém, como o VaR deve ser calculado diariamente para fins de exigência de capital, isto acarretaria em uma série de retornos fortemente autocorrelacionada.

\section{Aferição dos Métodos}

O Comitê da Basiléia argumenta que em épocas de crise a liquidez de muitos ativos pode diminuir sensivelmente, dificultando a alteração de posições em curtos períodos de tempo. Sugere, portanto, um prazo de dez dias durante o qual as perdas poderiam hipoteticamente acumular-se. Assim, a comparação se dá entre os retornos acumulados por dez dias pela carteira e a respectiva exigência de capital. A aferição engloba o período de 19/10/1995 a 31/7/2002, totalizando 1.675 observações.

Para o método padronizado verifica-se simplesmente o número de vezes em que as perdas de uma dada carteira superam a exigência de capital. A aferição dos métodos baseados em VaR segue as diretrizes constantes de documento publicado pelo Comitê da Basiléia especificamente com este propósito ${ }^{(13)}$. Desta forma, cada três meses verifica-se a quantidade de vezes em que, nos últimos 250 dias úteis, o VaR diário é superado pela perda verificada no dia, para cada carteira. Observe-se que, embora o VaR calculado para compor a exigência de capital seja baseado em um período de dez dias, a aferição do modelo interno utilizado se dá com base no VaR diário. O período aqui considerado originou 23 subperíodos de um ano. Basiléia estabelece um limite superior de quatro exceções em 250 observações. Entende-se por exceção a ocorrência de uma perda que ultrapasse a estimativa de VaR para o dia em estudo. Um número de exceções entre 5 e 10 pode levar, caso não haja forte justificativa para o desvio, a um aumento do multiplicador, que pode chegar a 4. Acima de 10 exceções, a instituição avaliada pode ser intimada a adotar a abordagem padronizada. Neste trabalho este conceito de exceção também é usado para uma perda acumulada de dez dias que ultrapasse a respectiva exigência de capital, embora o Comitê não preveja nenhum procedimento para a avaliação do desempenho do requerimento de capital. Assim, observa-se também o número de exceções da exigência de capital.

Vale observar que o procedimento de avaliação dos modelos (backtesting) recomendado pelo Comitê gera um comportamento inercial do padrão de exceções. Como as amostras têm 250 observações e são aferidas cada três meses, a interseção entre períodos adjacentes é de aproximadamente três quartos da amostra. Adicionalmente, no caso da exigência de capital, o fato de se 
considerar retornos acumulados de 10 dias úteis (holding period de 10 dias) pode gerar inércia do número de exceções constatado em dado período, na medida em que um retorno diário extremo tem seus efeitos perpetuados pelos dez dias úteis subseqüentes.

Adicionalmente, busca-se avaliar os métodos estudados com o auxílio do teste de Kupiec para percentual de exceções, aplicado ao período completo ${ }^{(14)}$. Neste trabalho, aplica-se às séries de projeções de VaR o teste de Kupiec para a proporção de exceções, com 5\% de nível de significância. Considerase o intervalo $(0,56 \% ; 1,51 \%)$ para a não rejeição da hipótese nula (H0) para a proporção empírica de vezes em que o VaR é ultrapassado, equivalente ao intervalo para exceções $(10 ; 25)$. Quando a proporção verificada está contida no intervalo especificado, H0 não é rejeitada. Adicionalmente calcula-se o pvalor para as proporções encontradas. Dado o nível de significância do teste, quanto maior for o p-valor em relação aos 5\%, maior a margem de segurança para a não rejeição de H0.

\section{Resultados}

Inicialmente apresenta-se, para os métodos baseados em VaR, os resultados da aplicação do teste de Basiléia e do teste de Kupiec ao VaR de um dia. Em seguida é examinado o desempenho da exigência de capital determinada por cada um dos métodos estudados, em face das perdas verificadas para ambas as carteiras.

\section{Desempenho do VaR de um Dia}

O VaR de um dia é analisado de duas formas. A primeira segue as orientações de Basiléia para backtesting, onde são registradas, cada três meses, as exceções ocorridas nos últimos 250 dias. Os resultados referentes a 23 subperíodos de 250 dias estão compilados na Tabela 2. A segunda consiste na aplicação do teste de Kupiec para a proporção de exceções ao período completo, conforme detalhado na Tabela 3. Os retornos diários e o VaR de um dia estão representado nos Gráficos 1 e 2. Os resultados são apresentados em duas seções, referentes a cada uma das carteiras I e II. 


\section{Tabela 2: Avaliação da Estimativa de VaR Diário por Subperíodos de 250 Dias}

\begin{tabular}{|c|c|c|c|c|c|c|c|c|c|c|}
\hline \multirow[b]{2}{*}{ Períodos / Métodos } & \multicolumn{5}{|c|}{ Exceções - Carteira I } & \multicolumn{5}{|c|}{ Exceções - Carteira II } \\
\hline & $\begin{array}{l}\text { Diag. } \\
\text { Simp. }\end{array}$ & $\begin{array}{l}\text { Diag. } \\
\text { Comp. }\end{array}$ & $\begin{array}{l}\text { Alis. } \\
0.94\end{array}$ & $\begin{array}{l}\text { Alis. } \\
0.90\end{array}$ & Hist. & $\begin{array}{l}\text { Diag. } \\
\text { Simp. }\end{array}$ & \begin{tabular}{|l|} 
Diag. \\
Comp.
\end{tabular} & $\begin{array}{l}\text { Alis. } \\
0.94\end{array}$ & $\begin{array}{c}\text { Alis. } \\
0.90\end{array}$ & Hist. \\
\hline 19/10/1995 a 22/10/1996 & 0 & 0 & 5 & 5 & 0 & 7 & 0 & 5 & 5 & 1 \\
\hline $22 / 01 / 1996$ a $23 / 01 / 1997$ & 0 & 0 & 3 & 2 & 0 & 11 & 0 & 4 & 4 & 1 \\
\hline 26/04/1996 a 29/04/1997 & 0 & 0 & 1 & 1 & 0 & 15 & 0 & 2 & 2 & 1 \\
\hline 26/07/1996 a 30/07/1997 & 4 & 4 & 3 & 3 & 4 & 24 & 4 & 5 & 4 & 5 \\
\hline $24 / 10 / 1996$ a $27 / 10 / 1997$ & 10 & 9 & 6 & 6 & 9 & 31 & 9 & 5 & 4 & 8 \\
\hline $28 / 01 / 1997$ a $28 / 01 / 1998$ & 13 & 12 & 7 & 6 & 11 & 32 & 12 & 6 & 5 & 9 \\
\hline 05/05/1997 a 05/05/1998 & 13 & 12 & 8 & 7 & 11 & 26 & 12 & 7 & 7 & 8 \\
\hline 04/08/1997 a 04/08/1998 & 9 & 8 & 8 & 8 & 7 & 14 & 7 & 4 & 5 & 4 \\
\hline $30 / 10 / 1997$ a $04 / 11 / 1998$ & 9 & 9 & 8 & 8 & 3 & 10 & 5 & 4 & 4 & 3 \\
\hline $02 / 02 / 1998$ a $08 / 02 / 1999$ & 7 & 7 & 8 & 9 & 3 & 8 & 3 & 4 & 4 & 4 \\
\hline 08/05/1998 a 13/05/1999 & 7 & 7 & 7 & 8 & 3 & 7 & 3 & 4 & 4 & 4 \\
\hline 07/08/1998 a 12/08/1999 & 7 & 7 & 5 & 5 & 3 & 7 & 3 & 4 & 4 & 4 \\
\hline $09 / 11 / 1998$ a $12 / 11 / 1999$ & 1 & 1 & 1 & 2 & 1 & 2 & 0 & 1 & 2 & 1 \\
\hline $11 / 02 / 1999$ a $15 / 02 / 2000$ & 0 & 0 & 1 & 1 & 1 & 4 & 1 & 2 & 4 & 1 \\
\hline $18 / 05 / 1999$ a $18 / 05 / 2000$ & 2 & 2 & 3 & 3 & 3 & 10 & 2 & 2 & 3 & 2 \\
\hline $17 / 08 / 1999$ a $16 / 08 / 2000$ & 3 & 2 & 3 & 4 & 3 & 15 & 2 & 2 & 3 & 2 \\
\hline $18 / 11 / 1999$ a $17 / 11 / 2000$ & 4 & 2 & 5 & 6 & 3 & 18 & 2 & 2 & 3 & 2 \\
\hline $18 / 02 / 2000$ a $20 / 02 / 2001$ & 5 & 3 & 5 & 8 & 3 & 16 & 1 & 2 & 2 & 1 \\
\hline $23 / 05 / 2000$ a $24 / 05 / 2001$ & 4 & 2 & 4 & 7 & 2 & 17 & 1 & 3 & 3 & 2 \\
\hline $21 / 08 / 2000$ a $23 / 08 / 2001$ & 3 & 2 & 4 & 7 & 2 & 19 & 2 & 4 & 5 & 3 \\
\hline $22 / 11 / 2000$ a $26 / 11 / 2001$ & 4 & 4 & 4 & 6 & 4 & 22 & 4 & 5 & 6 & 5 \\
\hline $23 / 02 / 2001$ a $04 / 03 / 2002$ & 3 & 3 & 3 & 4 & 3 & 24 & 5 & 5 & 6 & 6 \\
\hline $29 / 05 / 2001$ a $04 / 06 / 2002$ & 2 & 2 & 2 & 3 & 2 & 21 & 5 & 4 & 5 & 5 \\
\hline
\end{tabular}

Obs: o número de exceções é registrado em sub-períodos de 250 dias úteis a cada três meses. O Comitê de Basiléia sugere um limite máximo de 4 falhas para cada sub-período.

\section{Carteira I}

O VaR diário calculado com base no método diagonal simplificado apresenta mais de quatro exceções em nove dos 23 subperíodos analisados. As exceções de VaR concentram-se no período de julho de 1997 a setembro de 1998, que engloba as crises da Ásia e da Rússia ${ }^{(15)}$. Para a amostra completa o teste de Kupiec também rejeita o método com 31 exceções, seis acima do limite máximo definido. O desempenho do método diagonal completo é superior ao da sua forma simplificada. O número de subperíodos em que ocorrem mais de quatro exceções cai de nove para oito e o número total de exceções cai de 31 para 27, apenas duas acima do limite máximo permitido por Kupiec ${ }^{(16)}$.

Para o método baseado em alisamento exponencial com fator de alisamento $\lambda=0,94$ há mais de quatro exceções em 11 dos 23 subperíodos. Em muitos casos o número de exceções é próximo de quatro. Para toda a amostra, o teste de 
Kupiec também rejeita o método, com a ocorrência de 33 exceções. As exceções estão distribuídas ao longo do período estudado, ocorrendo alguma concentração no período compreendido entre outubro de 1997 e setembro de 1998. Apesar de estimado para o Ibovespa, o fator de alisamento $\lambda=0,90$ causa deterioração dos resultados em relação ao caso anterior. Há mais de quatro exceções em 14 dos 23 subperíodos. Para toda a amostra, o método apresenta 37 exceções, sendo rejeitado pelo teste de Kupiec.

O método histórico, dentre todos os estudados, apresenta o melhor desempenho para as estimativas de VaR, com apenas quatro subperíodos em que ocorrem mais de quatro exceções para a Carteira I. Contudo o afastamento do limite de 4 exceções é consideravelmente maior que o observado para o método baseado em alisamento exponencial (há 11 exceções em dois subperíodos) ${ }^{(17)}$. O melhor desempenho deste método se reflete no p-valor para o período completo $(0,1463)$, indicando que o método não pode ser rejeitado pelo teste de Kupiec.

\section{Tabela 3: Resumo dos Resultados do Teste Kupiec para os Métodos Diagonal, Baseado em Alisamento Exponencial e Histórico - Amostra Completa}

\begin{tabular}{|c|c|c|c|c|c|c|c|}
\cline { 2 - 8 } \multicolumn{2}{c|}{} & \multicolumn{3}{c|}{ Carteira I } & \multicolumn{3}{c|}{ Carteira II } \\
\hline \multirow{2}{*}{ Diagonal } & Métodos & Exceções & $\%$ & p-valor & Exceções & $\%$ & p-valor \\
\cline { 2 - 8 }$y$ & Simplificado & 31 & 1,85 & 0,00176 & 108 & 6,45 & 0,00000 \\
\hline $\begin{array}{c}\text { Baseado em } \\
\text { Alisamento } \\
\text { Exponencial }\end{array}$ & $\lambda=0,94$ & 33 & 1,97 & 0,00042 & 26 & 1,55 & 0,03560 \\
\hline & $\lambda=0,90$ & 37 & 2,21 & 0,00002 & 29 & 1,73 & 0,00643 \\
\hline
\end{tabular}

Obs: a coluna “p-valor” se refere ao teste de Kupiec com nível de significância de 5\%, aplicado apenas ao VaR de 1\%. Logo, p-valores iguais ou maiores que 0,05 indicam que o método de projeção do VaR passou no teste, ou seja, que a hipótese nula de que a verdadeira proporção de exceções é igual ao nível pré-especificado para o VaR (1\%) não pode ser rejeitada no nível de significância de 5\% do teste. A proporção de exceções é calculada para a amostra completa de 1.675 dias. 
Gráfico 1: Retornos Efetivos Diários e Estimativas de VaR Calculadas pelo Método Diagonal Completo, Alisamento Exponencial $(\lambda=0,94)$ e

Histórico para a Carteira I (Montante de $\mathbf{R} \$ \mathbf{1 0 0 . 0 0 0 , 0 0 )}$

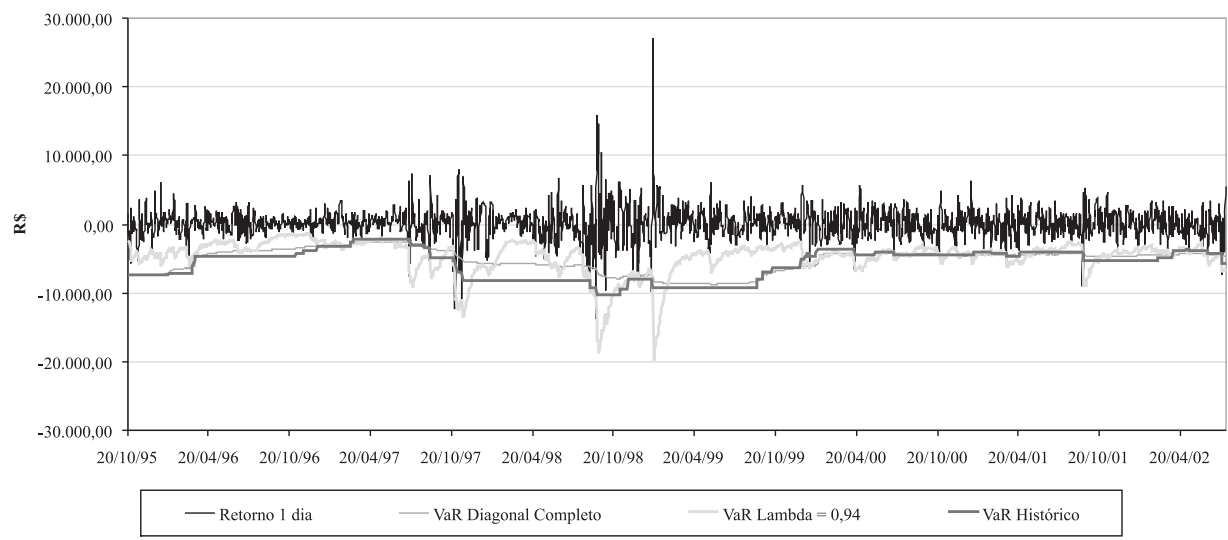

Gráfico 2: Retornos Efetivos Diários e Estimativas de VaR Calculadas pelo Método Diagonal Completo, Alisamento Exponencial $(\lambda=0,94)$ e Histórico para a Carteira II (Montante de $\mathbf{R} \$ \mathbf{2 0 . 0 0 0 , 0 0 )}$

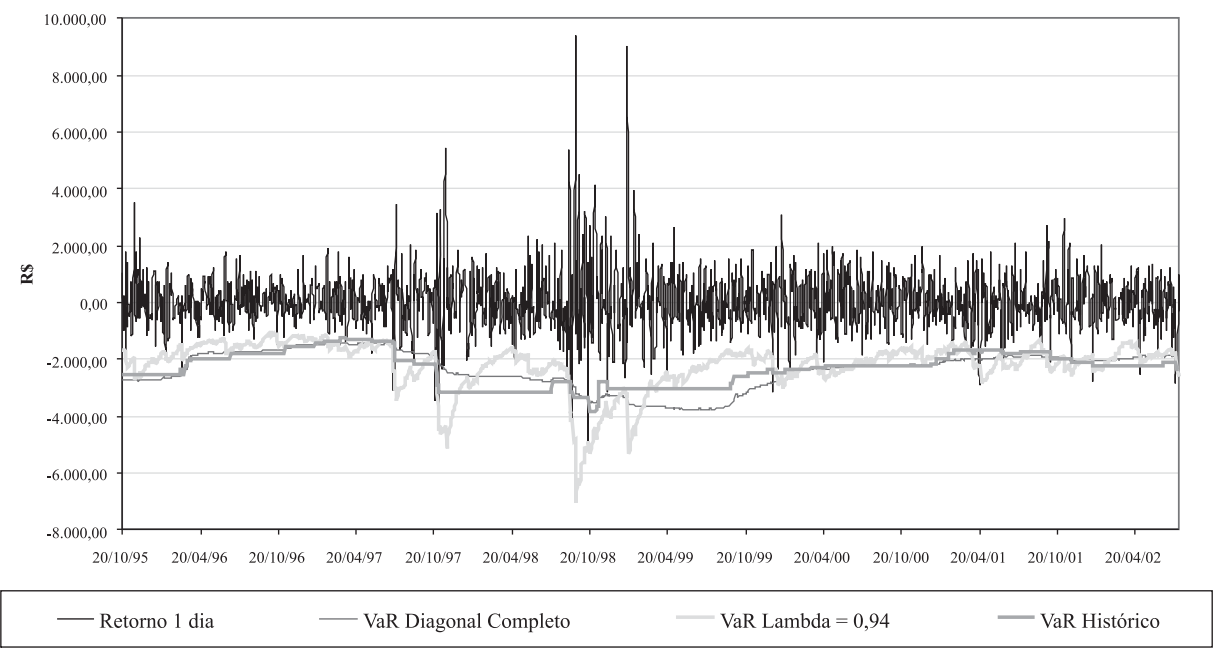

\section{Carteira II}

O método diagonal simplificado mostra um fraquíssimo desempenho, com mais de quatro exceções do VaR em 21 subperíodos. Para a amostra completa constatam-se 108 exceções (6,45\%), excedendo sobremaneira o limite máximo do teste de Kupiec. Este resultado sugere que o método simplificado pode 
apresentar sérios problemas, quando aplicado a carteiras com posições compradas e vendidas, que podem apresentar pesos expressivos para algumas ações. Para a versão completa do método diagonal, constata-se uma acentuada queda no número de exceções. Em apenas sete subperíodos há mais de quatro exceções e para a amostra completa ocorrem 25 exceções, de forma que o método não pode ser rejeitado pelo teste de $\operatorname{Kupiec}^{(18)}$.

O método de alisamento exponencial com fator 0,94 obtém melhores resultados para a Carteira II que para a I. Embora apresente mais de quatro exceções em 7 dos 23 subperíodos, os números estão sempre muito próximos deste limite. Para a amostra completa, o método é rejeitado ao ultrapassar o limite máximo por apenas uma exceção. Analogamente ao descrito para o fator 0,94 , o método com fator 0,90 obtém melhores resultados para a Carteira II que para a I, apresentando mais de quatro exceções em 8 subperíodos. Para a amostra completa o método é rejeitado por apresentar 29 exceções.

O método histórico mais uma vez detém o melhor desempenho para o VaR. O limite do teste de Basiléia é ultrapassado em sete subperíodos e, para a amostra completa, constatam-se 24 exceções, não ocorrendo rejeição pelo teste de Kupiec $^{(19)}$.

\section{Desempenho da Exigência de Capital}

Como na seção anterior, a análise do desempenho dos métodos no tocante à exigência de capital é apresentada em duas seções. A primeira seção se refere à carteira I, cujos resultados se encontram resumidos na Tabela 4 e representados nos Gráficos 3 e 4, enquanto a segunda seção é dedicada à carteira II, cujos resultados se encontram resumidos na Tabela 5 e representados nos Gráficos 5 e 6 . 
Tabela 4: Avaliação das Exceções para a Exigência de Capital (EC) para os Métodos Padronizado, Diagonal, de Alisamento Exponencial e Histórico para a Carteira I

\begin{tabular}{|c|c|c|c|c|c|c|c|c|c|}
\hline & & & & $\begin{array}{r}\text { Diferenç } \\
\mathrm{Su}\end{array}$ & $\begin{array}{l}\text { entre o EC } \\
\text { riores à } \mathrm{E}\end{array}$ & $\begin{array}{l}\text { e as Perdas } \\
(\%)\end{array}$ & $\begin{array}{l}\text { Difere } \\
\text { Perdas }\end{array}$ & $\begin{array}{l}\text { lças entre } \\
\text { Inferiores }\end{array}$ & $\begin{array}{l}\mathrm{EC} \mathrm{e} \mathrm{as} \\
\mathrm{EC}(\%)\end{array}$ \\
\hline \multicolumn{3}{|c|}{ Métodos } & Exceções & Média & Mínimo & Máximo & Média & Mínimo & Máximo \\
\hline \multirow{3}{*}{\multicolumn{2}{|c|}{ Padronizado }} & $\mathrm{EC}=8 \%$ & 35 & $4,14 \%$ & $0,00 \%$ & $13,71 \%$ & $11,71 \%$ & $0,14 \%$ & $15,98 \%$ \\
\hline & & $\mathrm{EC}=12 \%$ & 3 & $3,03 \%$ & $1,34 \%$ & $5,71 \%$ & $19,03 \%$ & $0,16 \%$ & $23,98 \%$ \\
\hline & & $\mathrm{EC}=15 \%$ & 0 & $0,00 \%$ & $0,00 \%$ & $0,00 \%$ & $24,94 \%$ & $0,29 \%$ & $29,98 \%$ \\
\hline \multirow{4}{*}{ Diagonal } & \multirow{2}{*}{ Simplificado } & $M=2$ & 5 & $2,32 \%$ & $0,15 \%$ & $5,51 \%$ & $26,36 \%$ & $0,47 \%$ & $52,33 \%$ \\
\hline & & $M=3$ & 0 & $0,00 \%$ & $0,00 \%$ & $0,00 \%$ & $41,76 \%$ & $4,77 \%$ & $78,53 \%$ \\
\hline & \multirow{2}{*}{ Completo } & $M=2$ & 2 & $3,47 \%$ & $3,08 \%$ & $3,86 \%$ & $28,16 \%$ & $0,86 \%$ & $54,53 \%$ \\
\hline & & $M=3$ & 0 & $0,00 \%$ & $0,00 \%$ & $0,00 \%$ & $44,64 \%$ & $7,24 \%$ & $81,84 \%$ \\
\hline \multirow{4}{*}{$\begin{array}{l}\text { Baseado em } \\
\text { Alisamento } \\
\text { Exponencial }\end{array}$} & \multirow{2}{*}{$\lambda=0,94$} & $M=2$ & 0 & $0,00 \%$ & $0,00 \%$ & $0,00 \%$ & $24,54 \%$ & $4,82 \%$ & $68,39 \%$ \\
\hline & & $M=3$ & 0 & $0,00 \%$ & $0,00 \%$ & $0,00 \%$ & $39,24 \%$ & $13,49 \%$ & $103,37 \%$ \\
\hline & \multirow{2}{*}{$\lambda=0,90$} & $M=2$ & 0 & $0,00 \%$ & $0,00 \%$ & $0,00 \%$ & $23,99 \%$ & $5,23 \%$ & $70,75 \%$ \\
\hline & & $M=3$ & 0 & $0,00 \%$ & $0,00 \%$ & $0,00 \%$ & $38,43 \%$ & $13,48 \%$ & $106,20 \%$ \\
\hline \multirow{2}{*}{\multicolumn{2}{|c|}{ Histórico }} & $M=2$ & 0 & $0,00 \%$ & $0,00 \%$ & $0,00 \%$ & $30,68 \%$ & $0,40 \%$ & $61,06 \%$ \\
\hline & & $M=3$ & 0 & $0,00 \%$ & $0,00 \%$ & $0,00 \%$ & $48,53 \%$ & $10,89 \%$ & $91,64 \%$ \\
\hline
\end{tabular}

Obs: a diferença entre a EC e a respectiva perda é calculada como percentual do montante de R\$ 100.000,00 da Carteira I.

\section{Carteira I}

O método padronizado mostra-se ineficiente para uma EC estipulada em 8\% do montante da carteira para cada risco (16\% no total), uma vez que acumula 35 exceções da exigência de capital em todo o período estudado. Com relação ao comportamento inercial do número de exceções, cabe enfatizar que a grande maioria das ocorrências se verifica entre janeiro de 1997 e junho de 1999, período que engloba as crises internacionais da Ásia e da Rússia, e a mudança do regime cambial brasileiro. Com efeito, 11 exceções são verificadas no curto período de pouco mais de duas semanas (27/10/97 a 12/11/97), em que a Bovespa sofreu fortes quedas ${ }^{(20)}$. Da mesma forma, 17 exceções ocorrem em menos de um mês $\left(12 / 8 / 98\right.$ a 10/9/98) ${ }^{(21)}$. Dado o fraquíssimo resultado, calcula-se a EC com os percentuais alternativos de $12 \%$ e $15 \%$ do montante da carteira ${ }^{(23)}$. São constatadas três e zero exceções, respectivamente. 
Gráfico 3: Retornos Efetivos de 10 Dias Úteis e Estimativas de EC, Calculadas pelo Método Padronizado, Método Diagonal Completo, Alisamento Exponencial $(\lambda=0,94)$ e Histórico para Carteira I (Montante de $\mathrm{R} \$ \mathbf{1 0 0 . 0 0 0 , 0 0 ) ,} M=2$

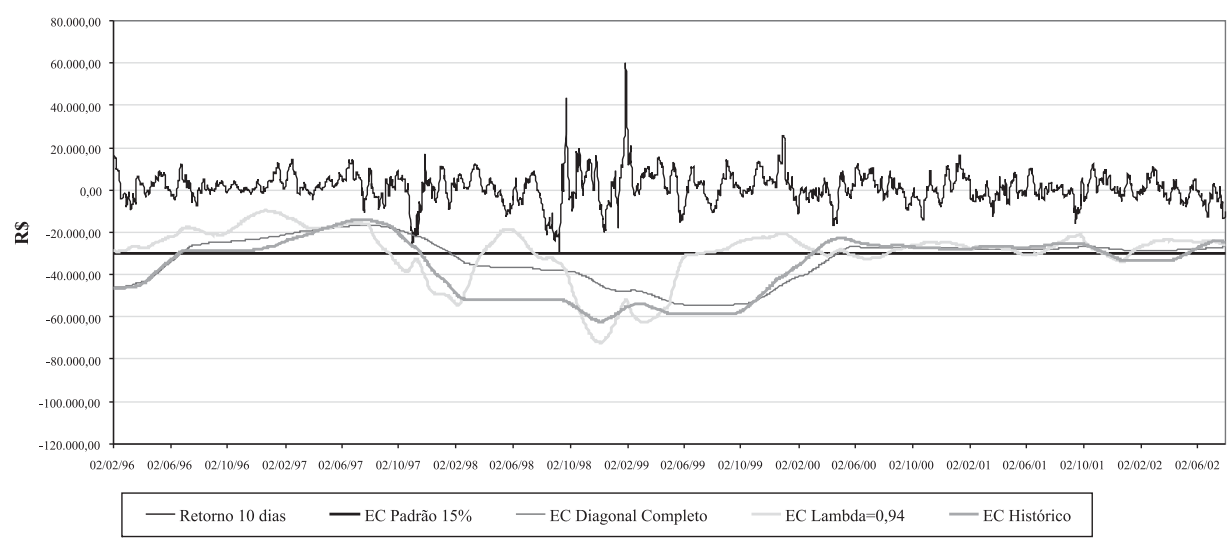

Gráfico 4: Retornos Efetivos de 10 Dias Úteis e Estimativas de EC, Calculadas pelo Método Padronizado, Método Diagonal Completo, Alisamento Exponencial $(\lambda=0,94)$ e Histórico para Carteira I (Montante de $\mathrm{R} \$ \mathbf{1 0 0 . 0 0 0 , 0 0 ) ,} M=3$

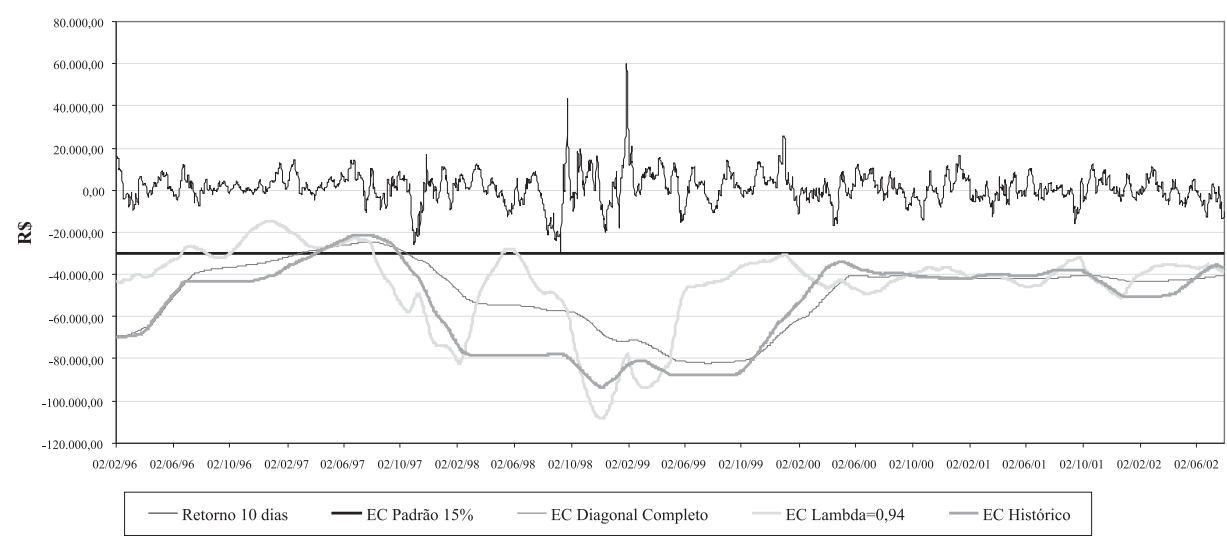

Para o método diagonal simplificado são registradas cinco exceções da exigência de capital para $\mathrm{M}=2$. Já o desempenho da versão completa do método diagonal é um pouco melhor, com duas exceções, também para $\mathrm{M}=2$.

O método de alisamento exponencial não apresenta exceções da exigência de capital para ambos os fatores de alisamento $(\lambda), 0,90$ e 0,94 , e para ambos os multiplicadores, 2 e 3 , o que pode indicar que o método permite um ajustamento eficaz para alterações na volatilidade. 


\section{Tabela 5: Avaliação das Exceções para a Exigência de Capital (EC) para os Métodos Padronizado, Diagonal, de Alisamento Exponencial e Histórico para a Carteira II}

\begin{tabular}{|c|c|c|c|c|c|c|c|c|c|}
\hline & & & & $\begin{array}{r}\text { Diferença } \\
\mathrm{Su}\end{array}$ & $\begin{array}{l}\text { entre o } \mathrm{EC} \\
\text { riores à } \mathrm{E}\end{array}$ & $\begin{array}{l}\text { as Perdas } \\
\text { (\%) }\end{array}$ & $\begin{array}{r}\text { Diferenças } \\
\text { Infe }\end{array}$ & $\begin{array}{l}\text { entre o EC } \\
\text { iores à } \mathrm{EC}\end{array}$ & $\begin{array}{l}\text { e as Perdas } \\
(\%)\end{array}$ \\
\hline \multicolumn{3}{|c|}{ Métodos } & Exceções & Média & Mínimo & Máximo & Média & Mínimo & Máximo \\
\hline \multirow{3}{*}{\multicolumn{2}{|c|}{ Padronizado }} & $\mathrm{EC}=8 \%$ & 0 & $0,00 \%$ & $0,00 \%$ & $0,00 \%$ & $37,64 \%$ & $2,44 \%$ & $47,97 \%$ \\
\hline & & $\mathrm{EC}=12 \%$ & 0 & $0,00 \%$ & $0,00 \%$ & $0,00 \%$ & $61,64 \%$ & $26,44 \%$ & $71,97 \%$ \\
\hline & & $\mathrm{EC}=15 \%$ & 0 & $0,00 \%$ & $0,00 \%$ & $0,00 \%$ & $79,64 \%$ & $44,44 \%$ & $89,97 \%$ \\
\hline \multirow{4}{*}{ Diagonal } & \multirow{2}{*}{ Simplificado } & $M=2$ & 8 & $5,44 \%$ & $0,71 \%$ & $9,60 \%$ & $41,56 \%$ & $0,37 \%$ & $89,12 \%$ \\
\hline & & $M=3$ & 0 & $0,00 \%$ & $0,00 \%$ & $0,00 \%$ & $66,81 \%$ & $4,47 \%$ & $133,82 \%$ \\
\hline & \multirow{2}{*}{ Completo } & $M=2$ & 0 & $0,00 \%$ & $0,00 \%$ & $0,00 \%$ & $65,15 \%$ & $11,28 \%$ & $118,19 \%$ \\
\hline & & $M=3$ & 0 & $0,00 \%$ & $0,00 \%$ & $0,00 \%$ & $102,90 \%$ & $37,28 \%$ & $177,37 \%$ \\
\hline \multirow{4}{*}{$\begin{array}{l}\text { Baseado em } \\
\text { Alisamento } \\
\text { Exponencial }\end{array}$} & \multirow{2}{*}{$\lambda=0,94$} & $M=2$ & 0 & $0,00 \%$ & $0,00 \%$ & $0,00 \%$ & $60,02 \%$ & $19,02 \%$ & $149,69 \%$ \\
\hline & & $M=3$ & 0 & $0,00 \%$ & $0,00 \%$ & $0,00 \%$ & $95,27 \%$ & $43,49 \%$ & $227,34 \%$ \\
\hline & \multirow{2}{*}{$\lambda=0,90$} & $M=2$ & 0 & $0,00 \%$ & $0,00 \%$ & $0,00 \%$ & $59,03 \%$ & $19,79 \%$ & $151,00 \%$ \\
\hline & & $M=3$ & 0 & $0,00 \%$ & $0,00 \%$ & $0,00 \%$ & $93,79 \%$ & $41,93 \%$ & $228,54 \%$ \\
\hline \multirow{2}{*}{\multicolumn{2}{|c|}{ Histórico }} & $M=2$ & 0 & $0,00 \%$ & $0,00 \%$ & $0,00 \%$ & $63,51 \%$ & $9,29 \%$ & $101,43 \%$ \\
\hline & & $M=3$ & 0 & $0,00 \%$ & $0,00 \%$ & $0,00 \%$ & $100,50 \%$ & $29,91 \%$ & $153,58 \%$ \\
\hline
\end{tabular}

Obs: a diferença entre a EC e a respectiva perda é calculada como percentual do montante de R\$ 20.000,00 da Carteira II.

Para o método histórico também não são verificadas exceções da EC, mas uma comparação dos valores relativos ao indicador [EC-perdas] na Tabela 4 permite inferir algumas diferenças relevantes. Observa-se que a diferença média entre a EC e as perdas na carteira [EC-perdas] é menor para o método de alisamento exponencial que para o histórico, o que configura uma menor alocação de capital para o primeiro método. Além disso, o método de alisamento exponencial possui a diferença mínima de [EC-perdas] maior que a do método histórico, o que indica que o método confere uma proteção maior sem excesso de alocação de capital. O Gráfico 1 sugere que este desempenho se deve ao fato de que a estimativa de VaR por este método acompanha mais de perto as alterações de volatilidade dos retornos.

\section{Carteira II}

Como todas as correlações dos ativos considerados neste trabalho são positivas, a carteira II tende a ter seu risco reduzido em relação à carteira I, por apresentar posições compradas e vendidas. O modelo padronizado reflete apenas em parte esta redução de risco, uma vez que o risco específico mantém como base de cálculo a soma dos módulos das posições. Isto explica em boa medida por que para a Carteira II já não há exceções com $8 \%$. 
O método diagonal simplificado é o único que apresenta exceções da exigência de capital (oito para $M=2$ ). $O$ fato de não se registrarem exceções para $M=3$ indica que as deficiências de um dado método de VaR podem ser encobertas por um multiplicador expressivo na determinação da EC. Já o desempenho da versão completa é nitidamente superior ao da versão simplificada, com zero exceções para ambos os multiplicadores.

Analogamente ao que ocorre com a carteira I, não há exceções na exigência de capital para o método de alisamento exponencial, para ambos os fatores de alisamento e para ambos os multiplicadores. O mesmo ocorre com o método histórico, que não registra exceções da EC para ambos os multiplicadores.

Mais uma vez, o método de alisamento exponencial possui as diferenças média e mínima de [EC-perdas], respectivamente, menor e maior que a do método histórico, o que sugere maior eficiência do método paramétrico, uma vez que ele fornece maior proteção sem excesso de alocação de capital (Tabela 5).

\section{Gráfico 5: Retornos Efetivos de 10 Dias Úteis e Estimativas de EC, Calculadas pelo Método Padronizado, Método Diagonal Completo, Alisamento Exponencial $(\lambda=0,94)$ e Histórico para Carteira II (Montante de $\mathbf{R} \$ \mathbf{1 0 0 . 0 0 0 , 0 0 ) ,} M=2$}

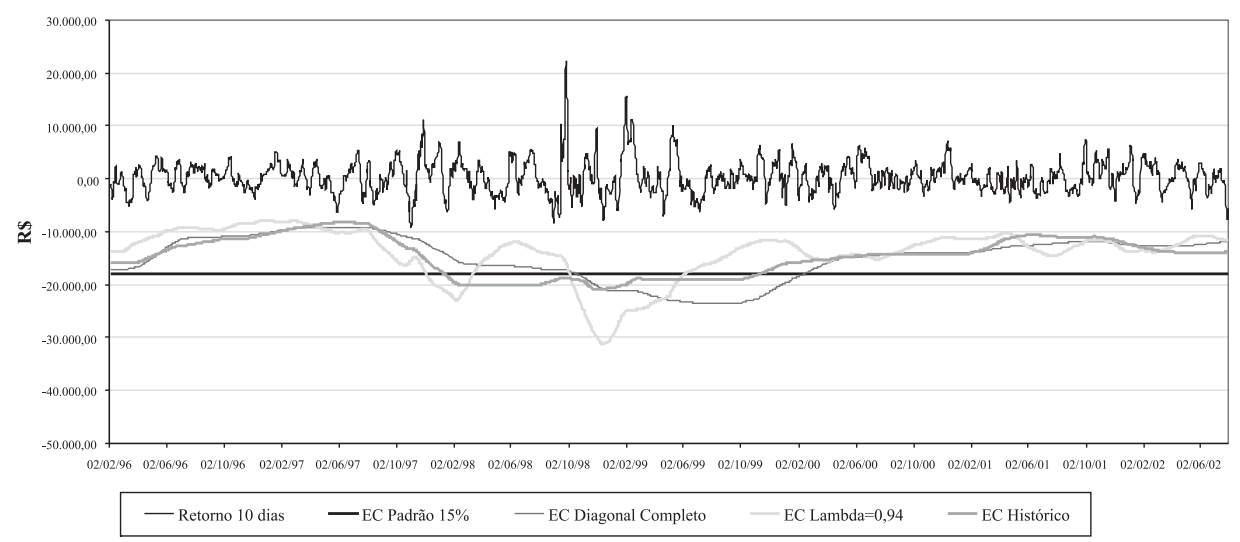


Gráfico 6: Retornos Efetivos de 10 Dias Úteis e Estimativas de EC, Calculadas pelo Método Padronizado, Método Diagonal Completo, Alisamento Exponencial $(\lambda=0,94)$ e Histórico para Carteira II (Montante de $\mathbf{R} \$ \mathbf{1 0 0 . 0 0 0 , 0 0 )}, M=3$

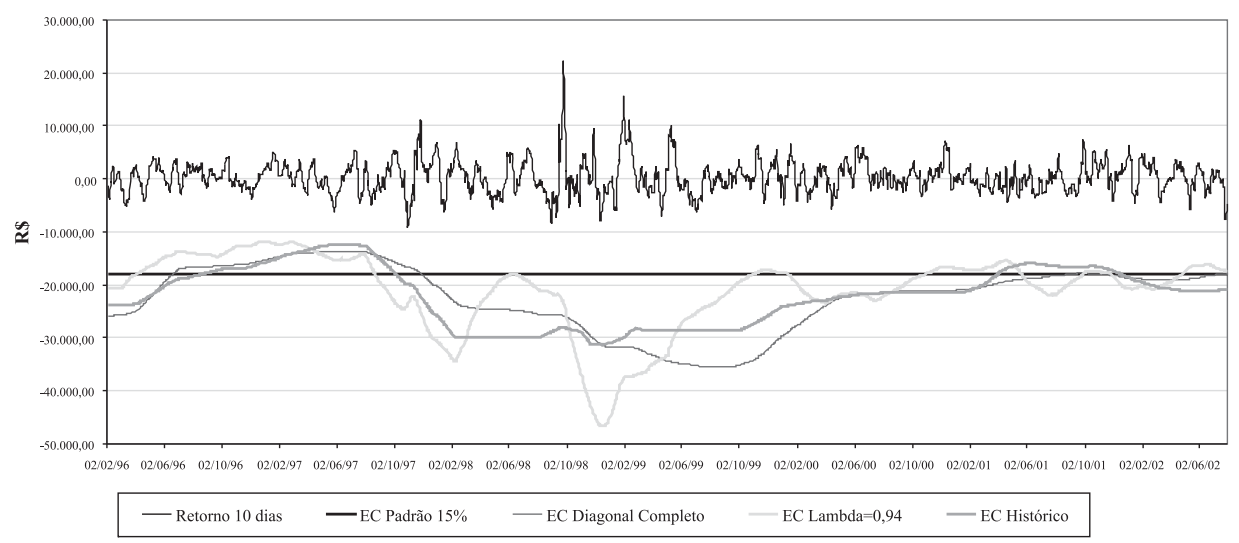

\section{Conclusões e Considerações Finais}

Os resultados alcançados podem ser resumidos nos seguintes pontos.

I. O método padronizado com EC igual ao percentual sugerido por Basiléia (8\% do montante da carteira para cada risco, específico e geral) mostra-se ineficiente para lidar com a volatilidade exibida pelo mercado acionário brasileiro. O menor percentual para o qual o método não exibe exceções da EC é de 15\%. Como este método gera uma EC constante, sempre haverá, potencialmente, momentos em que o risco será subestimado, assim como em muitas vezes a alocação de capital para cobertura de riscos estará muito acima do necessário. O não reconhecimento das alterações nos regimes de volatilidade e nos padrões de correlações entre os ativos é a principal crítica com relação a este método.

II. O método diagonal completo apresenta desempenho razoável para ambas as carteiras. Contudo os ajustes às oscilações da volatilidade ocorrem de forma lenta, o que é caracterizado pelos longos trechos planos observados nas linhas de VaR. Já a versão simplificada demonstra ser inadequada para carteiras com posições mistas (compradas e vendidas), em que há participação expressiva de algum ativo, o que desencoraja o seu uso para situações gerais. 
III. O método baseado em alisamento exponencial obtém os seus melhores resultados com um fator de decaimento $\lambda$ igual a 0,94 . O fator de 0,90 , estimado com base na série de retornos do Ibovespa, buscando melhor ajuste ao padrão de volatilidade do mercado brasileiro de ações, gera maior número de exceções para ambas as carteiras estudadas. Verifica-se a característica básica do método, que é a agilidade de ajuste às alterações da volatilidade, representada pelo perfil recortado do VaR de um dia. Este importante aspecto se traduz na inexistência de exceções da EC para ambas as carteiras, mesmo utilizando-se um multiplicador igual a dois e apesar de apresentar um desempenho insuficiente do VaR de um dia. Observe-se ainda que, para ambas as carteiras, a média das diferenças entre as exigências de capital calculadas e as perdas efetivamente verificadas [EC - perdas] é, para este método, a menor, considerando-se ambos os multiplicadores. Além disso, o valor mínimo deste indicador é sempre mais alto para este método. Portanto, mesmo com um requerimento médio de capital menor, a proteção oferecida é eficaz, o que sugere maior eficiência deste método.

IV. O método histórico é o único método, na abordagem de modelos internos, que não é rejeitado pelo teste de Kupiec para a amostra completa em ambas as carteiras. Da mesma forma que o método baseado em alisamento exponencial, não apresenta exceções da EC para ambas as carteiras. Contudo não aloca a exigência de capital com a mesma eficiência. Tal fato pode ser explicado pela baixa adaptabilidade do método às flutuações da volatilidade, o que pode ser identificado pelos longos platôs exibidos pelas linhas de VaR. Esta característica é conseqüência da metodologia empregada, que utiliza um dado percentil da amostra de retornos para VaR, o qual então se move em degraus, podendo manter-se inalterado por períodos relativamente longos de tempo. Vale, contudo, ressaltar a maior vantagem do método, que é a sua simplicidade e facilidade de implementação. Isto explica por que, apesar de limitado, é um método de VaR muito utilizado na prática.

Cabe lembrar que boa parte das exceções exibidas pelo VaR de um dia em todos os métodos ocorre em momentos de graves crises internacionais. Como todos os métodos se ressentem desses eventos, há que se ter maior cuidado em sua avaliação. Mesmo o Comitê de Basiléia prevê a possibilidade de que modelos internos que não atendam ao desempenho mínimo previsto (menos de quatro exceções do VaR diário em 250 dias úteis) não venham a ser automaticamente punidos. Pelo contrário, compete à autoridade reguladora avaliar as circunstâncias em que o fraco desempenho ocorreu, buscando detectar se o modelo realmente apresenta inconsistências em sua concepção. Em mercados emergentes tal avaliação deve ser extremamente criteriosa, uma vez que o VaR é reconhecidamente uma boa medida de risco para situações normais, mas é 
bastante questionado em situações que apresentem fortes variações do padrão de volatilidade, geralmente acompanhadas da ocorrência de valores extremos e de caudas pesadas na distribuição de retornos, o que ocorre com certa freqüência em mercados como o brasileiro.

Outro ponto importante surge ao se verificar que o método com pior desempenho nos testes para o VaR diário (método diagonal simplificado) não apresentou exceções de EC para $\mathrm{M}=3$, mesmo em períodos de crise. Isto pode sugerir que o multiplicador preconizado pelo Comitê para o cálculo da exigência de capital seja excessivo, quando aplicado ao mercado acionário brasileiro.

Neste sentido, o bom desempenho, em relação à exigência de capital, do método baseado em alisamento exponencial sugere que um método que se adapte rapidamente às mudanças da volatilidade pode permitir o uso de um multiplicador menor do que 3. Desta forma, seria possível manter ao mesmo tempo um nível de proteção adequado e atingir maior eficiência na alocação de capital. Este aspecto é reforçado pelo comportamento do método histórico, cuja adaptação às flutuações da volatilidade é lenta, em que o melhor desempenho no VaR diário é acompanhado de uma alocação menos eficiente do requerimento de capital.

Embora este trabalho tenha considerado apenas duas carteiras construídas de forma determinística, uma extrapolação desses resultados para outras carteiras pode ser considerada, quando contrapomos esses dois tipos de abordagem: a padronizada e a baseada em VaR. Em mercados voláteis, é desaconselhável o uso de métodos de cálculo de exigência de capital insensíveis ao risco. O uso desta abordagem só deve ser considerado no caso de carteiras de ações cuja participação na exposição geral da instituição financeira ao risco de mercado seja pouco relevante.

\section{Artigo recebido em 13.10.2003. Aprovado em 23.11.2004.}

\section{Notas}

${ }^{1}$ Ver Basle Committee on Banking Supervision (1996-A).

${ }^{2}$ Ver Banco Central do Brasil (1999-A, 1999-B, 2000-A, 2000-B e 2004).

${ }^{3}$ A Telemar PN apresenta participação de 13,58\% no Ibovespa em 30/7/2002. Em segundo lugar aparece a Petrobrás PN, com 9,16\%.

${ }^{4}$ Por razões metodológicas que serão explicitadas mais adiante, a Telebrás comporá as carteiras até 29/9/1999, embora a Telemar já fosse negociada há um ano. Vale observar que o Recibo de Carteira Selecionada de Ações Telebrás foi negociado com relativa liquidez até meados do ano 2000. 
${ }^{5}$ De acordo com Ceretta e Costa Jr. (2000), a maior parte dos benefícios advindos da diversificação seria obtida já com 12 ações. A partir de 18 ações, os benefícios adicionais são praticamente irrelevantes.

${ }^{6}$ Vale observar que o uso desta fórmula impõe uma questão. O valor do VaR foi calculado com base na série de retornos logarítmicos, por melhor se aproximarem estes da distribuição normal que os retornos efetivos (ver COSTA, 1998, p. 27-30). Contudo, este valor será usado como estimativa de um quantil da distribuição empírica de retornos efetivos. A alternativa seria trabalhar apenas com a série de retornos efetivos. Opta-se por manter os cálculos com base nos retornos logarítmicos.

${ }^{7}$ Ver Basle Committee on Banking Supervision (1996-A), Seção B.3 (c).

${ }^{8}$ Ver Jorion (1998, p. 154-156).

${ }^{9}$ Segundo Jorion (1998, p. 155).

${ }^{10}$ Metodologia desenvolvida pelo banco norte-americano J. P. Morgan. Ver Riskmetrics Group (1996).

${ }^{11}$ Ver Jorion (1998).

12 Ver Basel Committee on Banking Supervision, 1996-A, seção B4, item c.

${ }^{13}$ Basle Committee on Banking Supervision (1996-B).

${ }^{14}$ Ver Kupiec (1995).

${ }^{15}$ Onze exceções de VaR ocorrem no período de 14/7/1997 a 30/10/1997.

${ }^{16}$ Dez exceções de VaR ocorrem no período de 14/7/1997 a 30/10/1997.

${ }^{17}$ Dez exceções ocorrem no período de 14/7/1997 a 30/10/97.

${ }^{18}$ Dez exceções ocorrem no período de 15/7/1997 a 30/10/1997.

${ }^{19}$ Sete exceções ocorrem entre 15/7/1997 e 30/10/1997.

${ }^{20}$ Por exemplo, em 27/10/97 o Ibovespa caiu 14,98\%, enquanto as ações de Bradesco, Eletrobrás e Petrobrás caíram 13,15\%, 17,36\% e 18,68\%, respectivamente.

${ }^{21}$ Por exemplo, em 12/8/98 as ações de Eletrobrás e CSN caíram 10,20\% e 14,35\%, respectivamente. E em 27/8/98 as ações de Cemig, Eletrobrás e Petrobrás caíram 10,93\%, 13,64\% e 13,10\%, respectivamente.

${ }^{22}$ Para cada risco (específico e geral), ou seja, $24 \%$ e $30 \%$ no total, respectivamente. $15 \%$ foi o primeiro percentual arredondado para o qual não ocorreram exceções da EC.

\section{REFERÊNCIAS}

AMANTE, A. O.;

LEMGRUBER, E.F.

Uma avaliação empírica da adequação de capital definida pelo acordo da Basiléia para cobertura do risco de mercado de carteiras de renda variável no Brasil. Gestão de Risco e Derivativos - Aplicações no Brasil. Coleção COPPEAD de Administração. 1. ed. São Paulo: Atlas. 2001. p. 221-241. 
BANCO CENTRAL DO BRASIL.

Seção Legislação e Normas - Normas do CMN e do BC. Resolução $\mathbf{N}^{\mathbf{0}}$ 2.606, 27 maio 1999-A. Disponível em: $<$ http://www.bcb.gov.br. Acesso em: 17 jun. 2002.

Seção Legislação e Normas - Normas do CMN e do BC. Circular $\mathbf{N}^{\mathbf{2}} \mathbf{2 . 8 9 4}$, 27 maio 1999-B. Disponível em: <http:/ /www.bcb.gov.br. Acesso em: 17 jun. 2002.

Seção Legislação e Normas - Normas do CMN e do BC. Resolução $\mathbf{N}^{\mathbf{0}}$ 2.692, 24 fev. 2000-A. Disponível em: <http://www.bcb.gov.br. Acesso em: 17 jun. 2002.

Seção Legislação e Normas - Normas do CMN e do BC. Circular $\mathbf{N}^{\circ} \mathbf{2 . 9 7 2}$, 23 mar. 2000-B. Disponível em <http:/ /www.bcb.gov.br>. Acesso em: 17 jun. 2002.

Seção Legislação e Normas - Normas do CMN e do BC. Circular $\mathbf{N}^{0}$ 3.229, 25 mar. 2004. Disponível em <http:// www.bcb.gov.br>. Acesso em: 29 mar. 2004

\section{BASLE COMMITTEE ON BANKING SUPERVISION.}

Amendment to the capital accord to incorporate market risks, jan. 1996a. Disponível em: < http://www.bis.org/ bcbs>. Acesso em: $1^{\circ}$ jul. 2002.

Supervisory framework for the use of "Backtesting" in conjunction with the internal models aproach to market risk capital requirements, jan. 1996b. Disponível em: < http:// www.bis.org/bcbs>. Acesso em: $1^{\circ}$ jul. 2002.
CERETTA, P. S;

COSTA Jr., N. C. A.

Quantas ações tornam um portfólio diversificado no mercado de capitais brasileiro? Mercado de Capitais análise empírica no Brasil. Coleção COPPEAD de Administração, 1. ed. São Paulo: Atlas. 2000. p. 15-33.

COSTA, C. L.

Opções: operando a volatilidade, 1 . ed. São Paulo: BM\&F-Cultura, 1998.

\section{CROUHY, M.;}

GALAI, D.;

MARK, R.

The New 1998 Regulatory Framework for Capital Adequacy: "Standardized Approach" versus "Internal Models”. Net Exposure - The Eletronic Journa I of Financial Risk. Issue 4, jan. 1998. Disponível em: <http://www.netexposure.co.uk/>. Acesso em: 4 dez. 2002.

\section{HENDRICKS, D.;}

\section{HIRTLE, B.}

Bank Capital Requirements for Market Risk: the internal model approach. FRBNY Economic Policy Review, dec. 1997. Disponível em: <http://www.newyorkfed.org/ research/epr>. Acesso em: 4 dez. 2002.

\section{JORION, P.}

Value at Risk: a nova fonte de referência para o controle de risco de mercado. Bolsa de Mercadorias e Futuros, 1. ed. São Paulo, 1998.

\section{KUPIEC, P.}

Techniques for verifying the accuracy of risk measurement models. Journal of Derivatives, n. 2, p. 73-84, winter 1995. 
RISKMETRICS GROUP.

RiskMetrics - Technical Document.

New York - J. P. Morgan, 1996.

Disponível em: <http://

www.riskmetrics.com/techdoc.html>.

Acesso em: 26 nov. 2002. 